\title{
Role of goblet cell protein CLCA1 in murine DSS colitis
}

\author{
Nancy A. Erickson ${ }^{1}$, Lars Mundhenk', Samoa Giovannini ${ }^{1}$, Rainer Glauben², Markus M. Heimesaat ${ }^{3}$ and \\ Achim D. Gruber ${ }^{1 *}$
}

\begin{abstract}
Background: The secreted goblet cell protein CLCA1 (chloride channel regulator, calcium-activated-1) is, in addition to its established role in epithelial chloride conductance regulation, thought to act as a multifunctional signaling protein, including cellular differentiation pathways and induction of mucus production. Specifically, CLCA1 has recently been shown to modulate early immune responses by regulation of cytokines. Here, we analyze the role of CLCA1, which is highly expressed and secreted by colon goblet cells, in the course of murine dextran sodium sulfate-induced colitis.

Findings: We compared Clca1-deficient and wild type mice under unchallenged and DSS-challenged conditions at various time points, including weight loss, colon weight-length-ratio and histological characterization of inflammation and regeneration. Expression levels of relevant cytokines, trefoil factor 3 and E-cadherin were assessed via quantitative PCR and cytometric bead arrays. Lack of CLCA1 was associated with a more than two-fold increased expression of CxCl-1-and II-17-mRNA during DSS colitis. However, no differences were found between Clca1-deficient and wild type mice under unchallenged or DSS-challenged conditions in terms of clinical findings, disease progression, colitis outcome, epithelial defects or regeneration.
\end{abstract}

Conclusions: CLCA1 is involved in the modulation of cytokine responses in the colon, albeit differently than what had been observed in the lungs. Obviously, the pathways involved depend on the type of challenge, time point or tissue environment.

Keywords: $\mathrm{ClCa}^{\text {\% }}$, CXCL-1, Keratinocyte chemottractant, CXCL-2, MIP-2a, Inflammation, Signaling molecule, IL-17, E-cadherin, TFF3

\section{Introduction}

The goblet cell-derived protein CLCA1 (chloride channel regulator, calcium-activated 1) is thought to act as a multifunctional signaling protein via as yet unidentified molecular pathways. Originally, CLCA1 had been thought to modulate epithelial cell chloride conductance. Subsequent work has shown, however, that it may also induce airway mucus production through an interleukin (IL)-13-mediated cascade [1] and promote spontaneous differentiation while reducing proliferation of Caco-2 cells [2]. In a similar context, CLCA1 expression was proposed as a prognostic factor in colorectal cancer $[2,3]$.

\footnotetext{
* Correspondence: achim.gruber@fu-berlin.de

'Department of Veterinary Pathology, Freie Universität Berlin,

Robert-von-Ostertag-Strasse 15, 14163 Berlin, Germany

Full list of author information is available at the end of the article
}

Recently, CLCA1 was shown to modulate pulmonary cytokine expression in early immune responses, specifically the pro-inflammatory response of human airway macrophages in vitro [4], whereas more complex results have been obtained from mouse models [5, 6]. In Clca1deficient $\left(\mathrm{Clca1}^{-1-}\right)$ mice, experimental Staphylococcus (S.) aureus pneumonia was associated with decreased responses of chemokine (C-X-C motif) ligand (CXCL)-1, a potent neutrophil chemoattractant, with consequently decreased neutrophil recruitment [5]. Furthermore, lack of Clca1 expression yielded reduced responses of the pro-inflammatory cytokine IL-17. In contrast, following intranasal ovalbumin or lipopolysaccharide (LPS) challenge, Clca1-deficiency resulted in increased neutrophil recruitment preceded by CXCL-1 upregulation in the LPS model [6]. Thus, the role of CLCA1 in cytokine 
modulation seems to be complex and dependent on the stimulus used.

Here, we investigated the proposed function of CLCA1 in modulating the early immune response in the colon, the tissue in which CLCA1 is most highly expressed in man [7] and mice [8]. We chose the dextran sodium sulfate (DSS) challenge model which is commonly used to study early immune reactions in mouse intestine [9]. In a previous DSS colitis study, we failed to observe any effects of Clca1-deficiency on mucus barrier integrity and mucin gene expression [10] which likely would have affected secondary immune responses. Additionally, recent studies have indicated that CLCA1 does not play a role in calcium-activated chloride secretion in the respiratory tract nor does restoration of reduced Clcal expression rectify the cystic fibrosis electrophysiology defect in the intestine $[11,12]$. In light of the absence of such possibly interfering effects, we postulated that possible differences in inflammatory parameters during DSS colitis in the $\mathrm{Clca1}^{-/-}$model would be due to primary CLCA1 effects on the immune response. We thus compared $\mathrm{Clcal}^{-1-}$ and wild type (WT) mice under unchallenged and DSS-challenged conditions in terms of key clinical and histopathological parameters as well as expression profiles of select cytokines. Furthermore, we determined expression levels of goblet cell-derived trefoil factor (Tff)-3, a key regulator in mucosal repair and protection [13], and E-cadherin (E-cad) which is down-regulated after Clca1-knock-down in vitro [3].

\section{Materials and methods}

Ethics statement, mice and DSS treatment

$\mathrm{Clcal}^{-1-}$ and WT mice were given $2.5 \%$ DSS for 24 (24 h-group), $48 \mathrm{~h}$ (48 h-group) or for 7 days with 2 consecutive days of water (7 d-group) as described [10]. For ethics statement, selection and treatment see Additional file 1.

\section{Weight loss, colon weight-length-ratio and sampling}

In the $7 \mathrm{~d}$-group, weight loss was determined in the course of DSS administration and colon weight-lengthratio at necropsy. Colons of all groups were equally sectioned from proximal to distal for histopathology which were immediately fixed in $4 \%$ buffered formalin. For Reverse Transcriptase-quantitative PCR (RT-qPCR) and organ culture, sections were opened longitudinally, flushed with ice-cold Dulbecco's phosphate buffered saline (biowest, Nuaille, France) and either snap frozen in liquid nitrogen and stored at $-80{ }^{\circ} \mathrm{C}$ or immediately processed, respectively.

\section{Histopathology}

$4 \mu \mathrm{m}$ thick formalin-fixed, paraffin-embedded, hematoxylin and eosin-stained sections of proximal and distal colon were evaluated separately by veterinary pathologists in a blinded fashion according to a scoring scheme (Additional file 2).

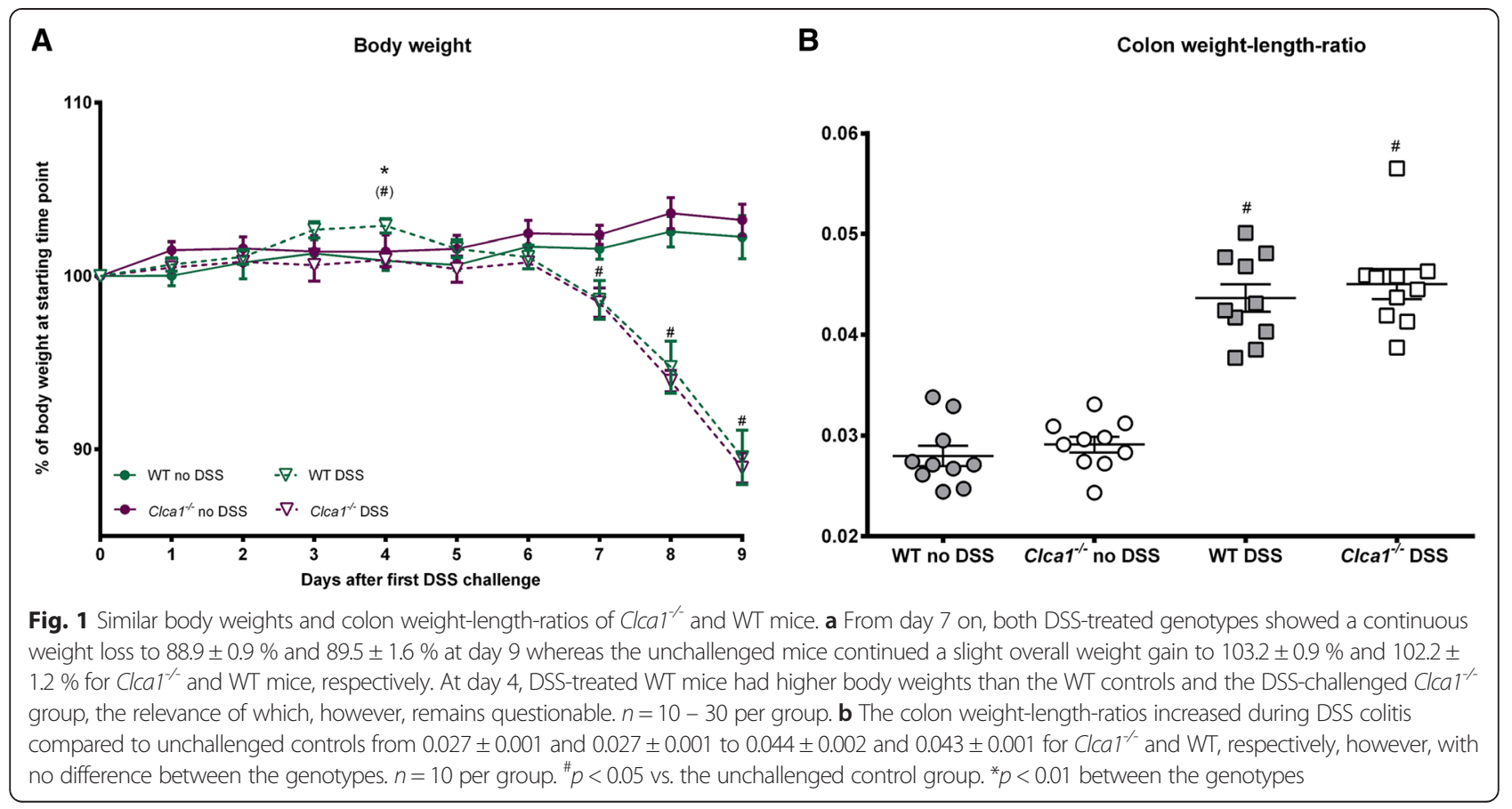




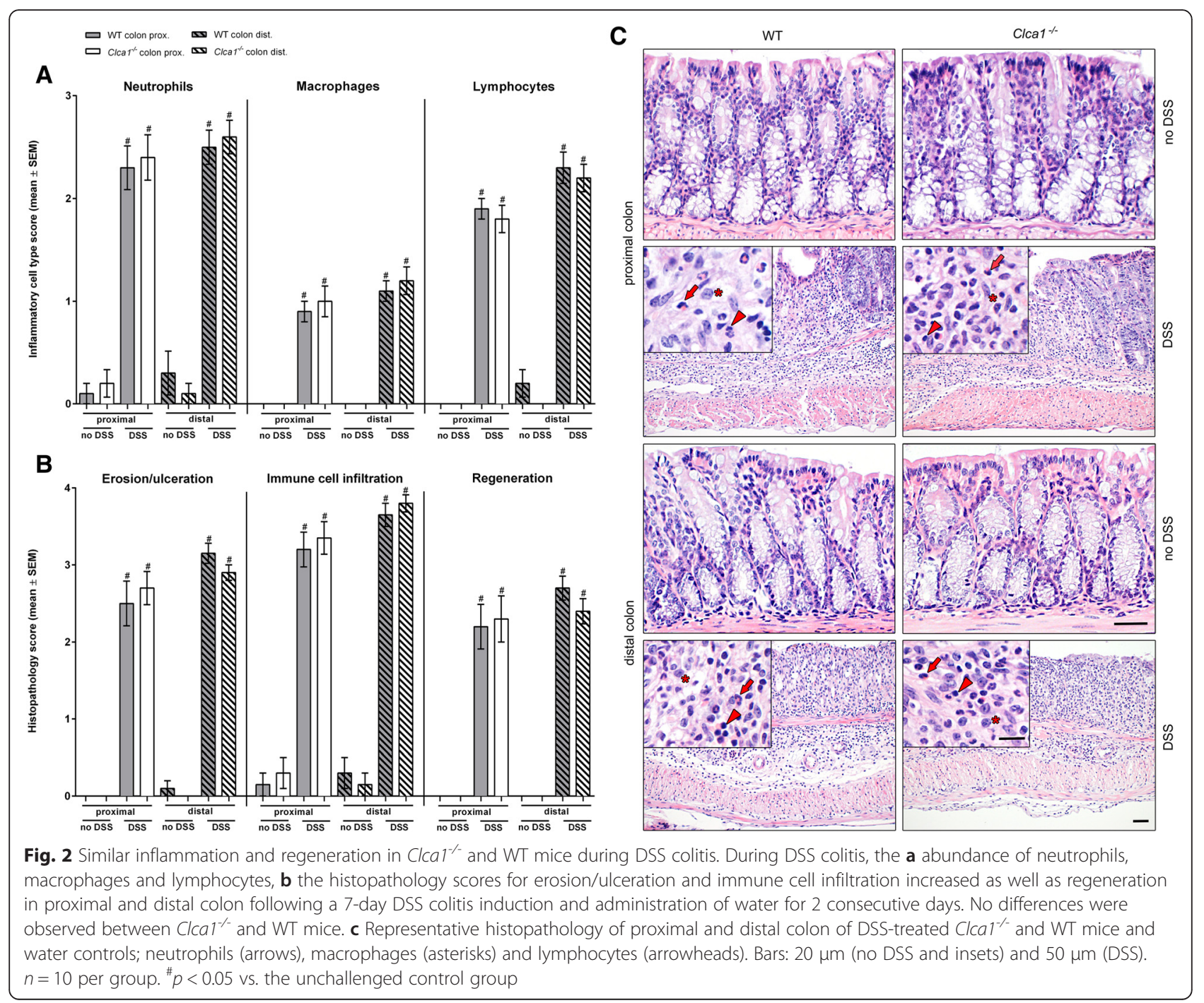

\section{RNA isolation and reverse transcriptase-qPCR}

Total RNA isolation, primer and probe design, RT-qPCR and data analysis were performed as described $[5,10]$. Transcript expression levels of Cxcl-1, Cxcl-2, Il-17, Tnf, Ifny, Tff3 and E-cad were determined and normalized to the internal reference genes glyceraldehyde-3-phosphate

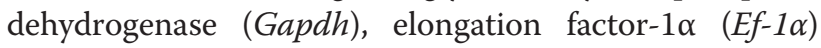
and $\mathrm{B}-2$ microglobulin $(B 2 m)$ as described [5]. Primers and probes are listed in Additional file 3.

\section{Cytometric bead array}

Colon cultures were established and supernatants processed as described [14] and stored at $-80{ }^{\circ} \mathrm{C}$ until further analysis. Cytokine concentrations of CXCL-1, monocyte chemoattractant protein (MCP)-1, TNF, IFN $\gamma$, IL-1 $\beta,-2$, $-6,-13$ and $-17 \mathrm{~A}$ were determined via cytometric bead array using a FACSCantoII and the FacsDiva software (all BD Biosciences, Heidelberg, Germany) as described $[15,16]$.

\section{Statistics}

Statistical analyses via Mann-Whitney-U test and graphical illustrations were performed using GraphPad PRISM 6 (GraphPad Software Inc., La Jolla, USA) and data are expressed as mean \pm standard error of the mean (SEM) except for RT-qPCR data. Here, data are expressed as single value fold change of which a more than two-fold difference between the genotypes was considered relevant.

\section{Results and discussion}

The percentile body weights declined following day 6 of DSS application (Fig. 1a) and colon weight-length-ratios increased (Fig. 1b), without any difference between the genotypes (Fig. 1) which is in line with the genotypeindependent decrease of stool consistency and increase of fecal blood content in the DSS colitis model described earlier [10]. At day 4, DSS-treated WT mice had higher body weights compared to the DSS-challenged $\mathrm{Clcal}^{-1-}$ 


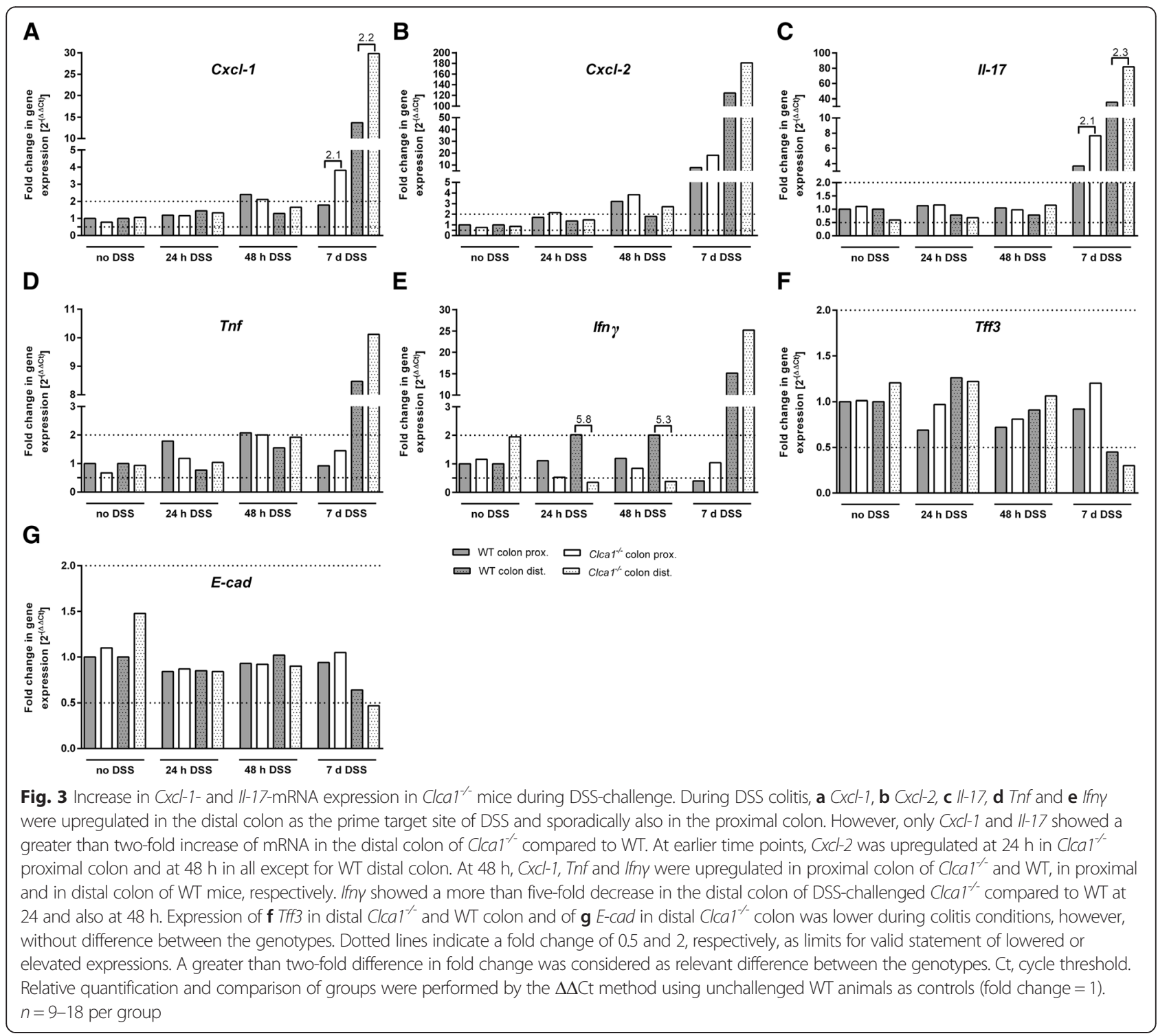

mice but also to unchallenged WT controls. In this context and being only $2 \%$ in difference between the genotypes, this single statistically significant data point is biologically questionable. Alternatively, in the context of Ifny mRNA decrease at early time points (see below) in $\mathrm{Clcal}^{-1}$ mice, this may indicate changes in metabolism in Clca1-deficient mice only. Importantly, Clca1deficiency had no impact on any clinical parameter tested which is in line with the previous respiratory challenge model [5].

Histopathologically, Clca1-deficiency did not result in any effect on the extent and nature of inflammatory cells, epithelial defects and regeneration. During DSS colitis, neutrophils, macrophages and lymphocytes (Fig. 2a and c, insets) as well as erosion/ulceration, immune cell infiltration and regeneration (Fig. 2b and c) increased in proximal and distal colon, clearly reflecting the expected inflammation. At 24 und $48 \mathrm{~h}$, no significant immune cell infiltrations or histopathologic alterations were observed (Additional file 4). In contrast to previous studies on airway inflammation in which neutrophil responses were either decreased in $\mathrm{Clcal}^{-/-}$mice after $S$. aureus infection [5] or increased after LPS challenge [6], no difference was observed histopathologically in the colitis model between the genotypes.

As expected, mRNA expressions of Cxcl-1, Cxcl-2, Il17, Tnf and Ifny (Fig. 3a to e) were overall upregulated in distal and, sporadically, also in proximal colon during DSS colitis. These cytokines, except for Il-17, occasionally were slightly elevated at earlier time points.

However, $\mathrm{Cxcl}-1$ and $\mathrm{Il}-17$ (Fig. 3a and c) showed a greater than two-fold increase of mRNA copy numbers in distal colon of $\mathrm{Clcal}^{-/}$compared to WT mice during colitis. The significance of the transiently lower Ifny 


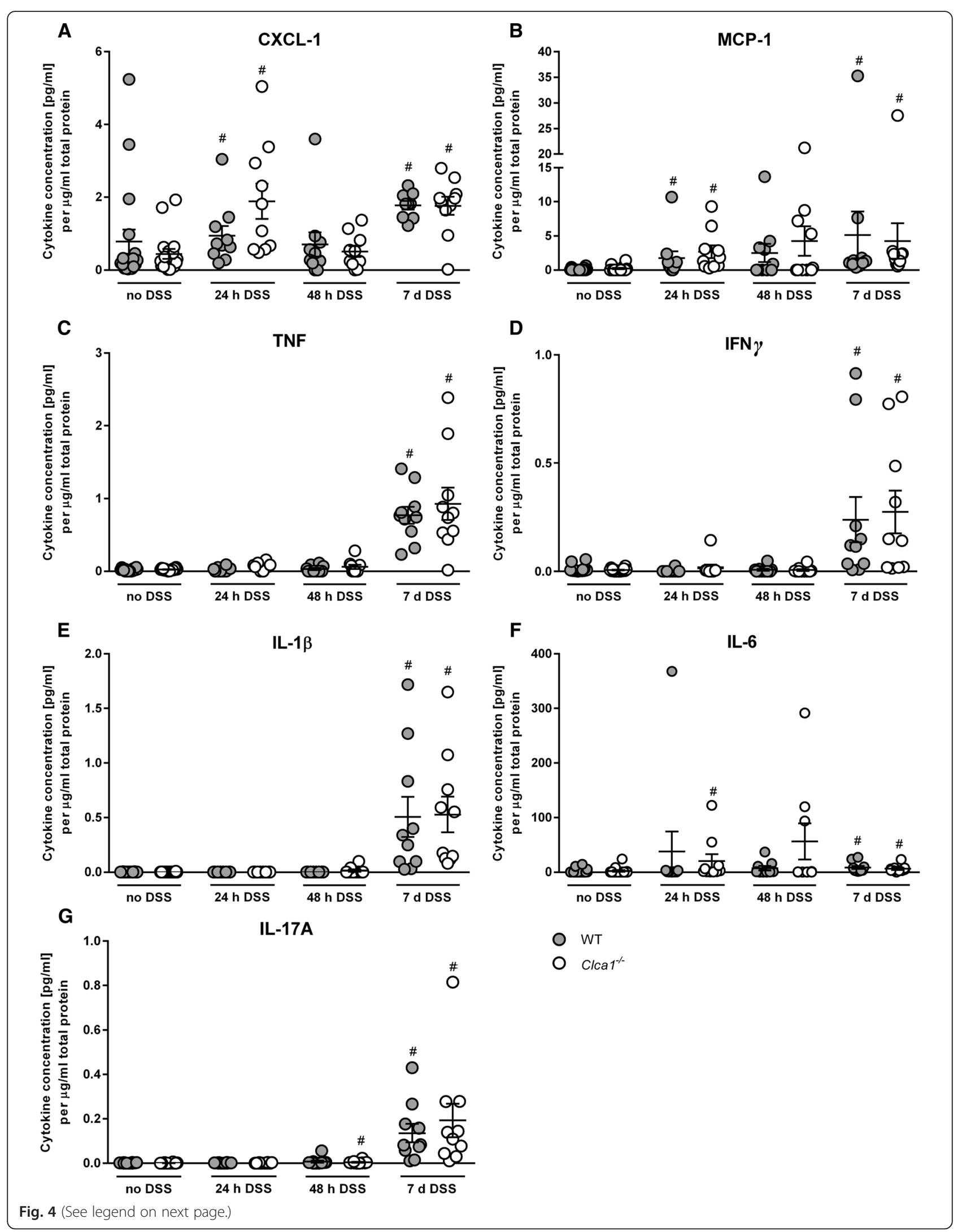


(See figure on previous page.)

Fig. 4 Inflammatory cytokines are similarly upregulated on protein level in DSS colitis between the genotypes. Cytokines were determined on the protein level in colon supernatant via cytometric bead array and normalized to the respective total protein concentration. a CXCL-1, b MCP-1, $\mathbf{c}$ $T N F$, $\mathbf{d} I F N \gamma, \mathbf{e} I L-1 \beta, \mathbf{f} \| L-6$ and $\mathbf{g} I L-17 A$ were elevated during DSS colitis without any difference between the genotypes. CXCL-1, MCP-1 and IL-6, the latter only in $\mathrm{ClCal}^{-1-}$, seemed also slightly elevated at $24 \mathrm{~h}$ as well as IL-17A in Clcal ${ }^{-1-}$ at $48 \mathrm{~h}$ after DSS challenge, however, without statistical significance between the genotypes. ${ }^{*} p<0.05$ vs. the unchallenged control group. $n=9-18$ per group. Only if more than 5 mice had detectable protein values, the group was considered for valid statement of significance

expression levels in the distal colon of DSS-challenged $\mathrm{Clcal}^{-1-}$ compared to WT at 24 and $48 \mathrm{~h} \mathrm{(Fig.} \mathrm{3e)} \mathrm{is} \mathrm{un-}$ clear due to lack of histologically evident immune cell infiltration at these time points (Additional file 4). Expression of Tff3 mRNA in distal colon of $\mathrm{Clcal}^{-/}$and WT mice and of E-cad in distal $\mathrm{Clca1}^{-1-}$ colon (Fig. 3f and $\mathrm{g}$ ) was lower during colitis, likely due to destruction of goblet and epithelial cells, respectively, without differences between the genotypes.

CXCL-1, MCP-1, TNF, IFN $\gamma$, IL-1 $\beta,-6$ and -17A proteins (Fig. 4a to g) were similarly elevated during DSS colitis, CXCL-1 and MCP-1 also slightly at $24 \mathrm{~h}$ of DSSchallenge in both genotypes, IL-6 at 24 and IL-17A at $48 \mathrm{~h}$ in the $\mathrm{Clcal}^{--}$mice.

The cytokines IL-2 and -13 were below the detection limits at all time points.

Interestingly, differential expression of Cxcl-1 mRNA has also been found in respiratory Clca1deficient mouse models [5], consequentially with differences in CXCL-1 protein level and neutrophil recruitment $[5,6]$. Additionally, $I l-17$ was also differentially expressed in one model [5]. The prominently higher mRNA expression levels of $\mathrm{Cxcl}-1$ and $\mathrm{Il}-17$ in $\mathrm{Clcal}^{-/-}$DSS colitis mice may point towards initial regulatory events. This may become obvious on protein level at later time points as had been seen in the $S$. aureus pneumonia model [5]. The increase (1.4 fold) of IL-17A in the $\mathrm{Clcal}^{-1}$ mice of the $7-\mathrm{d}$ group may confirm this notion.

Despite being opposite to the $S$. aureus pneumonia model in which $\mathrm{Cxcl}-1$ and $\mathrm{Il}-17$ were decreased in $\mathrm{Clca1}^{-1-}$ mice [5], the DSS colitis data are in line with increased CXCL-1 responses following respiratory LPSchallenge [6]. Early Clcal-linked immune response modulation therefore seems to depend on the stimulus used. As solely LPS-mediated Toll-like receptor 4 signaling seems to be important for neutrophil recruitment, control of bacterial translocation and epithelial repair in acute DSS colitis [17], this model possibly shares similarities with the respiratory LPS challenge.

Our findings confirm the link of CLCA1 to early immune response modulation with a specific effect on Cxcl-1 and Il-17 albeit not decisive for clinical outcome. Potentially overlapping effects of CLCA1, including anion conductance and cellular differentiation, will have to be taken into account when further deciphering the interaction of Clca1 with $\mathrm{Cxcl-1}$ and $\mathrm{Il-17}$ in early immune responses.

\section{Additional files}

Additional file 1: Ethics statement, selection and treatment. (PDF $34 \mathrm{~kb}$ )

Additional file 2: Histopathological scoring scheme. (PDF $80 \mathrm{~kb}$ ) Additional file 3: Quantitative RT-PCR - primer and probe sequences. (PDF $108 \mathrm{~kb}$ )

Additional file 4: Results of histopathology scoring of 24 and 48 h-groups. (PDF $81 \mathrm{~kb}$ )

\section{Abbreviations}

CLCA: Chloride channel regulator, calcium-activated; gob-5: Goblet cell protein-5; DSS: Dextran sodium sulfate; $\mathrm{ClCa1}^{-1-}$ : Clca1-deficient; Cxcl: Chemokine (C-X-C motif) ligand; IL: Interleukin; LPS: Lipopolysaccharide; WT: Wild type; RT-qPCR: Reverse transcriptase quantitative polymerase chain reaction; Tff: Trefoil factor; IFNY: Interferone gamma; FELASA: Federation of Laboratory Animal Science Associations; h: Hours; d: Days; mRNA: Messenger ribonucleic acid; TNF: Tumor necrosis factor; Gapdh: Glyceraldehyde-3phosphate dehydrogenase; Ef-1a: Elongation factor 1alpha; B2m: beta-2 microglobulin; MCP: Monocyte chemoattractant protein; SEM: Standard error of the mean; pg: Picograms; $\mu$ g: Micrograms; ml: Milliliters; $S$.

aureus: Staphylococcus aureus; KC: Keratinocyte chemoattractant; vs.: Versus; MIP-2a: Macrophage inflammatory protein 2-alpha; E-cad: E-cadherin (alias (dh1).

\section{Competing interests}

The authors declare that they have no competing interests.

\section{Authors' contributions}

NAE designed and executed the experiments, analyzed the data and wrote the paper. LM conceived of, designed and partially executed the experiments, provided critical analysis and reviewed the manuscript. SG partially executed the data analysis. RG partially executed the experiments and provided essential expertise. MMH provided essential expertise, participated in the study design and reviewed the manuscript. ADG conceived of and coordinated the study, participated in its design, provided critical expertise and reviewed the manuscript. All authors read and approved of the final manuscript.

\section{Acknowledgements}

The exceptional technical assistance of Jana Enders and Inka Freise is greatly appreciated. We acknowledge Stefan Bereswill and André Fischer for providing laboratory equipment and reagents. This work is part of the doctoral thesis of Nancy A. Erickson and was supported by the German Research Foundation (DFG) SFB633, TP B6 (to MMH).

\section{Author details}

${ }^{1}$ Department of Veterinary Pathology, Freie Universität Berlin, Robert-von-Ostertag-Strasse 15, 14163 Berlin, Germany. ${ }^{2}$ Medical Department, Division of Gastroenterology, Infectiology and Rheumatology, Charité Universitätsmedizin Berlin, Hindenburgdamm 30, 12200 Berlin, Germany. ${ }^{3}$ Department of Microbiology and Hygiene, Charité - Universitätsmedizin Berlin, Garystrasse 5, 14195 Berlin, Germany. 
Received: 22 September 2015 Accepted: 27 January 2016

Published online: 04 February 2016

\section{References}

1. Alevy YG, Patel AC, Romero AG, Patel DA, Tucker J, Roswit WT, et al. IL-13induced airway mucus production is attenuated by MAPK13 inhibition. J Clin Invest. 2012;122:4555-68.

2. Yang B, Cao L, Liu B, McCaig CD, Pu J. The transition from proliferation to differentiation in colorectal cancer is regulated by the calcium activated chloride channel A1. PLoS One. 2013;8:e60861.

3. Yang B, Cao L, Liu J, Xu Y, Milne G, Chan W, et al. Low expression of chloride channel accessory 1 predicts a poor prognosis in colorectal cancer. Cancer. 2015;121:1570-80.

4. Ching JC, Lobanova L, Loewen ME. Secreted hCLCA1 is a signaling molecule that activates airway macrophages. PLoS One. 2013;8:e83130.

5. Dietert K, Reppe K, Mundhenk L, Witzenrath M, Gruber AD. mCLCA3 modulates IL-17 and CXCL-1 induction and leukocyte recruitment in murine Staphylococcus aureus pneumonia. PLoS One. 2014;9:e102606.

6. Long AJ, Sypek JP, Askew R, Fish SC, Mason LE, Williams CM, et al. Gob-5 contributes to goblet cell hyperplasia and modulates pulmonary tissue inflammation. Am J Respir Cell Mol Biol. 2006;35:357-65.

7. Gruber AD, Elble RC, Ji HL, Schreur KD, Fuller CM, Pauli BU. Genomic cloning, molecular characterization, and functional analysis of human CLCA1, the first human member of the family of $\mathrm{Ca} 2+$-activated $\mathrm{Cl}$ channel proteins. Genomics. 1998;54:200-14.

8. Leverkoehne I, Gruber AD. The murine mCLCA3 (alias gob-5) protein is located in the mucin granule membranes of intestinal, respiratory, and uterine goblet cells. J Histochem Cytochem. 2002;50:829-38.

9. Wirtz S, Neufert C, Weigmann B, Neurath MF. Chemically induced mouse models of intestinal inflammation. Nat Protoc. 2007;2:541-6.

10. Erickson NA, Nystrom EE, Mundhenk L, Arike L, Glauben R, Heimesaat MM, et al. The Goblet Cell Protein Clca1 (Alias mClca3 or Gob-5) Is Not Required for Intestinal Mucus Synthesis, Structure and Barrier Function in Naive or DSS-Challenged Mice. PLoS One. 2015;10:e0131991.

11. Mundhenk L, Johannesson B, Anagnostopoulou P, Braun J, Bothe MK, Schultz C, et al. mCLCA3 does not contribute to calcium-activated chloride conductance in murine airways. Am J Respir Cell Mol Biol. 2012;47:87-93.

12. Young FD, Newbigging $S$, Choi C, Keet M, Kent G, Rozmahel RF. Amelioration of cystic fibrosis intestinal mucous disease in mice by restoration of mCLCA3. Gastroenterology. 2007;133:1928-37.

13. Podolsky DK, Gerken G, Eyking A, Cario E. Colitis-associated variant of TLR2 causes impaired mucosal repair because of TFF3 deficiency. Gastroenterology. 2009;137:209-20.

14. Heimesaat MM, Dunay IR, Fuchs D, Trautmann D, Fischer A, Kuhl AA, et al. The distinct roles of MMP-2 and MMP-9 in acute DSS colitis. Eur J Microbiol Immunol (Bp). 2011;1:302-10.

15. Batra A, Heimesaat MM, Bereswill S, Fischer A, Glauben R, Kunkel D, et al. Mesenteric fat - control site for bacterial translocation in colitis? Mucosal Immunol. 2012;5:580-91.

16. Glauben R, Batra A, Fedke I, Zeitz M, Lehr HA, Leoni F, et al. Histone hyperacetylation is associated with amelioration of experimental colitis in mice. J Immunol. 2006;176:5015-22.

17. Fukata M, Michelsen KS, Eri R, Thomas LS, Hu B, Lukasek K, et al. Toll-like receptor-4 is required for intestinal response to epithelial injury and limiting bacterial translocation in a murine model of acute colitis. Am J Physiol Gastrointest Liver Physiol. 2005;288:G1055-1065.

\section{Submit your next manuscript to BioMed Central and we will help you at every step:}

- We accept pre-submission inquiries

- Our selector tool helps you to find the most relevant journal

- We provide round the clock customer support

- Convenient online submission

- Thorough peer review

- Inclusion in PubMed and all major indexing services

- Maximum visibility for your research

Submit your manuscript at www.biomedcentral.com/submit
C Biomed Central 